\title{
Reducing Patient Financial Liability for Hospitalizations: The Physician Role
}

Edward A. Ross, mo ${ }^{1}$

Frank B. Bellamy, RN, $\mathrm{MSN}^{2}$

\author{
${ }^{1}$ Division of Nephrology, Hypertension, and Transplantation, University of Florida, Gainesville, Florida. \\ ${ }^{2}$ Department of Case Management, Shands Hospital at the University of Florida, Gainesville, Florida.
}

Disclosure: Authors have no conflicts of interest.

With increasingly strict guidelines for insurance coverage, hospitals have adopted meticulous resource utilization review and management processes. It is important for physicians to appreciate that careful documentation of certain patient parameters may not only optimize the facility's reimbursement but have profound impact on the patient's out-of-pocket expenses. Hospital utilization teams have access to the frequently changing national payor guidelines for policy benefits, usually revolving around whether the patient meets medical necessity criteria for being classified as an "inpatient" vs. an "observation" outpatient. Those statuses are not merely time-based, and lead to marked differences in patient deductibles and coverage for medication, room, procedure, laboratory, and ancillary charges. There are nationally-recognized guidelines for classification, based on severity of illness and intensity of services provided. By participating in case management activities, physicians can have an important patient advocate role, and thereby minimize the financial burden to these individuals and their families. Journal of Hospital Medicine 2010;5:160-162. ○ 2010 Society of Hospital Medicine.

KEYWORDS: billing and compliance systems, communication systems, cost effectiveness systems, resident education.

Hospitalizations often impose a tremendous financial burden on patients and their families, adding to the stress and long-term impact from medical illnesses. It is widely underappreciated that physicians can play an important role in substantially reducing patients' out-of-pocket expenses by participating in hospital-based case review and utilization management. These topics are not a focus of most formal training curricula and unfortunately are typically viewed by medical staff as intrusive, time consuming, or only in terms of enhancing the facility's profitability. In reality, with strict rules governing insurance benefits the facility's interests are typically aligned with those of the patient.

One of the greatest impacts on a patient's financial liability is whether an admission is classified in "observation" vs. "inpatient" status, and is subject to much confusion. It is a common misperception that these are time-based designations. Instead, they revolve around stringent medical necessity guidelines that examine the severity of the illness and the intensity of services provided. ${ }^{1}$ Inpatient stays may be brief, even a single day, if justified by medical need (although these short durations are closely scrutinized by the payors) or if involving a short list of procedures automatically triggering that status (ie, defibrillator placement). ${ }^{2}$ Conversely, observation status, although usually up to only 48 hours, can extend longer if inpatient criteria are never met and are then apt to generate large bills.

The key concept for the financial liability of patients in observation status is that their billing structure revolves around being categorized as "outpatients," even though they stay overnight and are physically housed and cared for in the expensive hospital setting. ${ }^{3}$ This nonintuitive classifi- cation can culminate in unexpectedly high charges for which the patient is liable (Table 1): medications at inflated hospital pharmacy prices, especially when expensive antibiotics or immunosuppressive agents are administered (since outpatient prescriptions are not often covered by policies); ancillary services, radiology or laboratory tests with a high patient share of cost; and an hourly room charge that can easily exceed $\$ 30$ per hour. The latter can be especially burdensome, as most insurance plans only cover the first 48 hours of observation. During that period the patient would be liable for just their copayment, but afterward they could be billed for the full amount. Hospitalizations well beyond the 48 hours can thus present tremendous hardships to those patients who never meet the stringent criteria for categorization as inpatients, and whose status thus must remain outpatient-observation. Keeping patients over a weekend for procedures that are not available at the facility until the following Monday can put these individuals beyond the 48 hour observation interval and cause unintentional rapidly escalating out-of-pocket expenses. Other strategies to reduce the patient's financial liability include allowing patients to take their own medications from home (with pharmacy supervision and verification, per hospital guidelines), and limiting evaluations to just the admitting diagnosis (ie, pursuing other issues after discharge). In addition, an observation stay can never be ordered ahead of time for an outpatient procedure, as that type of admission is reserved for those individuals who unexpectedly need further care at the conclusion of the recovery period (typically 4 to 6 hours). Thus, the not uncommon practice of "doing a patient a favor" by letting them stay overnight after an outpatient 


\begin{tabular}{|c|c|c|}
\hline & $\begin{array}{l}\text { Observation (Outpatient Status) } \\
\text { (Medicare Part B) }\end{array}$ & $\begin{array}{l}\text { Inpatient } \\
\text { (Medicare Part A) }\end{array}$ \\
\hline Room and board & & $\begin{array}{l}\text { Medicare deductible: } \$ 1068 \text { per } \\
\text { admission (waived if } \\
\text { readmission in }<60 \text { days) }\end{array}$ \\
\hline$\leq 48$ hours & $20 \%$ of allowable charge (APC) & \\
\hline$>48$ hours & $100 \%$ hospital charges & \\
\hline Medications & $100 \%$ hospital charges & \\
\hline Supplies & Up to $100 \%$ hospital charges & \\
\hline \multicolumn{3}{|l|}{ Surgical } \\
\hline Operating room & Typically $20 \%$ copay of APC & \\
\hline Recovery room & Typically $20 \%$ copay of APC & \\
\hline \multicolumn{3}{|c|}{ Diagnostic } \\
\hline Laboratory & $20 \%$ copay of allowable charges & \\
\hline Radiology & $20 \%$ copay of allowable charges & \\
\hline \multicolumn{3}{|l|}{ Ancillary } \\
\hline Physical therapy & $20 \%$ copay of allowable charges & \\
\hline $\begin{array}{l}\text { Occupational } \\
\text { therapy }\end{array}$ & $20 \%$ copay of allowable charges & \\
\hline Speech therapy & $20 \%$ copay of allowable charges & \\
\hline
\end{tabular}

procedure thereby can be a great disservice by dramatically increasing patient liability. One can well imagine how these scenarios lead to lay press exposés of the patient receiving a bill for a \$25 aspirin and a night's stay 4 times more expensive than a luxury hotel. This is not to say that going home is the best or safest plan for a particular patient, but rather that the hospital is often an unnecessarily expensive (and in that sense inappropriate) location when there are alternatives. It is up to the individual hospital to determine how to handle rapidly escalating charges related to the admission status and the timeliness of a discharge. Many centers in effect "write off" highly select bills that are considered either uncollectible (ie, from indigent patients) or the fault of the facility's inefficiencies. So as not to have inconsistent billing policies across different insurers and patients, however, facilities are obligated to have uniform protocols for attempting to collect charges - a scenario that can be quite harsh for those individuals with significant and discoverable monetary resources.

Working with the physician for a timely discharge, hospital case managers and social workers are likely to arrive at creative solutions in the patient's best financial interest (ie, taxicab coupons and inexpensive hotels). As many patients simply do not have the resources to cope with unplanned overnight charges, it behooves the physician to make every effort to start outpatient procedures early in the day so as to minimize the chance of logistic problems triggering a potentially expensive overnight hospital stay.

Compare the observation patient's liability to that of the typically much-preferred status of inpatient (Table 1) in

\begin{tabular}{|c|c|c|}
\hline & $\begin{array}{l}\text { Patient Liability } \\
\text { for Observation } \\
\text { Status (Medicare Part B) }\end{array}$ & $\begin{array}{l}\text { Inpatient Charges } \\
\text { (Covered by Medicare } \\
\text { Part A Deductible) }\end{array}$ \\
\hline Room and board & $\$ 1788$ & $\$ 1030$ \\
\hline Medications & $\$ 755$ & $\$ 1196$ \\
\hline Supplies & $\$ 106$ & $\$ 528$ \\
\hline $\begin{array}{l}\text { Procedures and } \\
\text { emergency room }\end{array}$ & $\$ 229$ & $\$ 1145$ \\
\hline \multicolumn{3}{|l|}{ Diagnostic } \\
\hline Laboratory & $\$ 72$ & $\$ 359$ \\
\hline Radiology & $\$ 159$ & $\$ 795$ \\
\hline \multicolumn{3}{|l|}{ Ancillary } \\
\hline Electrocardiogram & $\$ 22$ & $\$ 110$ \\
\hline Physical therapy & $\$ 295$ & $\$ 1475$ \\
\hline $\begin{array}{l}\text { Patient liability for } \\
\text { hospitalization }\end{array}$ & $\$ 3426$ & $\begin{array}{l}\$ 1068 \text { deductible for } \\
\text { total charges of } \$ 6638\end{array}$ \\
\hline $\begin{array}{l}\text { Patient liability for } \\
\text { subsequent skilled } \\
\text { nursing facility }\end{array}$ & $\$ 159$ per day* & Small daily co-pay* \\
\hline
\end{tabular}

which all expenses are rolled into one diagnosis-related group (DRG) prospective payment. ${ }^{3}$ In the case of Medicare, the patient's bill would be the inpatient deductible, and this might be covered in its entirety by a supplemental policy. One absolutely cannot, however, simply avoid using the observation status and instead make all admissions "inpatients"; this would cause unnecessary resource utilization and expose the hospital to denial of payment for the entire episode of care. To prevent this situation, there are nationally-recognized guidelines that strictly define when a hospitalization warrants an inpatient level of care. Integral to the individual qualifying for their policy's inpatient benefit, however, is that the chart must reflect not just the "severity of illness" but also "intensity of services" ordered by the physician. ${ }^{1}$ Similarly, changing a patient's status (ie, from observation to inpatient) must follow rigorous guidelines wherein the justification and timing are fully described in the body of the chart to an extent that would withstand audit.

Consider the example (Table 2) of a patient with a leg fracture admitted for pain due to edema and early compartment syndrome: a scenario appropriate for inpatient status, liability of just the $\$ 1092$ Medicare deductible, and eligibility for postdischarge skilled nursing facility care. Had the charting erroneously only indicated pain and need for a new cast, then observation status would have yielded a bill for $\$ 3426$, plus out of pocket nursing home expenses of over $\$ 150 /$ day.

Not only does the physician need to accurately chart the reasons for admission, but it is also extremely helpful to specifically document why the patient is not amenable to 
outpatient therapy. Examples include a clearly articulated history of failed attempts at home or emergency room treatment, or the need for close monitoring (ie, telemetry). In this regard case managers also provide a fresh set of eyes to evaluate the clarity and completeness of medical charting. What seems like obvious decision-making to a physician may require expanded detailed notes to satisfy a third-party review.

The work design of the case managers and utilization review team varies between facilities. Ideally, cases are reviewed upon admission (or within the first 24 hours), and then periodically thereafter. Many medical centers have this process computerized, wherein inpatient criteria are available online and status issues can be tracked daily. This nearly real-time information serves as the basis for interacting with the attending physician, and is necessary because the chart documentation may not be amended after discharge. Having a robust database for all admissions is also immensely helpful in those hospitals which employ a "Physician Advisor" (PA) as a liaison and educator to the medical staff. This newly and now nationally recognized PA position serves an important role in educating the providers not just about these patient advocacy topics, but also other issues such as length-of-stay. Interestingly, having the infrastructure of a criteria-driven database to follow the intensity of inpatient services on a daily basis gives case managers an objective perspective of when a patient requires less care and is ready for transfer to a lower acuity facility or discharge home. Physician participation is important when the patient thus "runs out of intensities," since there will need to be early coordination of efforts for home health or skilled nursing care, durable medical equipment supplies, or outpatient infusions. It is important that physicians not view these activities as an inappropriate rush for discharge. In our experience most patients are in fact much happier to be out of the hospital and receiving home or skilled nursing care. Those in need of physical or occupational therapy may in fact have superior care in facilities dedicated to those activities. In addition, unnecessarily prolonged hospitalizations carry their own risks, such as hospital-acquired infections and deep venous thromboses. An additional motivator for discharge is that, just as there are insurance plan limits for outpatient benefits, there can also be caps for inpatient services. Physicians thus have a role in preserving the limited and precious number of covered inpatient days of care, beyond which time the patient would be financially totally responsible. For example, most states limit the number of inpatient days covered by Medicaid. In Florida there is a cap of only 45 days per year (unless the patient is pediatric or within the first year of a transplant ${ }^{4}$ ). Similarly, there have been patients and families shocked and ill-prepared to discover that all their Medicare hospital benefits were exhausted $^{5}$ : a not well-publicized possibility, as in the setting of expensive intensive care units, transplantation, or chemotherapy. Timely discharges and careful resource utilization by physicians thus not only help the hospital but also are important for the patient.

In summary, physicians need to be aware that there can be tremendous financial hardship to patients caused by inappropriate or unnecessarily long observation stays, especially in cases where an inpatient designation would have been justified by appropriate documentation. Case managers, although employed by the facility, can thus assist physicians in this regard and together play an important role as patient advocates.

\section{Address for correspondence and reprint requests:}

Edward A. Ross, MD, Physician Advisor, Shands Hospital at the University of Florida, Division of Nephrology, Hypertension \& Transplantation, University of Florida, Box 100224, Gainesville, FL 32610-0224; Telephone: 352-273-8822; Fax: 352-392-5465; E-mail: rossea@medicine.ufl.edu Received 17 June 2009; revision received 4 August 2009; accepted 29 August 2009.

\section{References}

1. Interqual ${ }^{B}$ Level of Care Criteria: Acute Care Adult. Newton, MA: McKesson Health Solutions; 2009.

2. Department of Health and Human Services (DHHS), Centers for Medicare and Medicaid Services (CMS). 2008. Final changes to the hospital outpatient prospective payment system and CY 2009. Available at: http:// www.cms.hhs.gov/HospitalOutpatientPPS/HORD/list.asp. Accessed September 2009.

3. Department of Health and Human Services (DHHS), Centers for Medicare and Medicaid Services (CMS). 2008. Medicare Program; Changes to the hospital inpatient prospective payment systems and fiscal year 2009 rates. Available at: http://www.cms.hhs.gov/AcuteInpatientPPS/IPPS/itemdetail.asp. Accessed September 2009.

4. Florida Medicaid covered services. Available at: http://www.fdhc.state. fl.us/Medicaid/MediPass/pdf/HealthyOutcomesCoveredServicesFlyerEnglish 0606.pdf. Accessed September 2009.

5. Medicare Benefit Policy Manual: Chapter 5. Lifetime reserve days. Available at: http://www.cms.hhs.gov/manuals/Downloads/bp102c05.pdf. Accessed September 2009. 